Research Article

Edwin Koźniewski and Anna Borowska*

\title{
Hyperboloid offset surface in the architecture and construction industry
}

https://doi.org/10.1515/eng-2019-0051

Received May 21, 2019; accepted Jul 02, 2019

\begin{abstract}
In this paper the issue of approximation of the hyperboloid offset surface off $(S(t, v) ; d)$ at distance $d$ by the hyperboloid surface $S_{1}(\phi, v)$ is considered. The problem of determining various surfaces approximating the hyperboloid offset surface off $(S(t, v) ; d)$ is important due to the applications of the hyperboloid as a mathematical model for miscellaneous objects in the architecture and construction industry. The paper presents the method of determining the angles and coordinates of points of various surfaces approximating the hyperboloid of revolution. A twosheet hyperboloid offset surface can be used for modelling double-layer domes. A one-sheet hyperboloid offset surface was used to model the reinforced structure of the cooling tower.
\end{abstract}

Keywords: hyperbola offset curve, one-sheet hyperboloid, two-sheet hyperboloid

\section{Introduction}

A one-sheet hyperboloid is often used as a model for various objects in the construction industry. It is a doubly ruled surface. Thanks to this, one-sheet hyperboloid shaped constructions can be built with, for example straight steel elements that form a strong structure. Such a clever design guarantees lower costs than other technical solutions. Examples of such constructions are cooling towers (Jaworzno, Cracow in Poland), Kobe Port Tower in Japan, Newcastle International Airport in England, Cathedral of Brasilia in Brazil, the Canton Tower in China and many other structures. A two-sheet hyperboloid can be used to model domes and other roofs which cover stadiums, halls,

\footnotetext{
^Corresponding Author: Anna Borowska: Faculty of Computer Science, Bialystok University of Technology, ul. Wiejska 45a, 15-351 Bialystok, Poland; Email: a.borowska@pb.edu.pl

Edwin Koźniewski: Faculty of Civil and Environmental Engineering, Bialystok University of Technology, ul. Wiejska 45e, 15-351 Bialystok, Poland; Email: e.kozniewski@pb.edu.pl
}

and shopping centres. Nowadays, lattice domes with elaborate shapes (Golden Terraces in Warsaw) and multilayer (retractable) roofs of large objects (National Stadium in Singapore) are used.

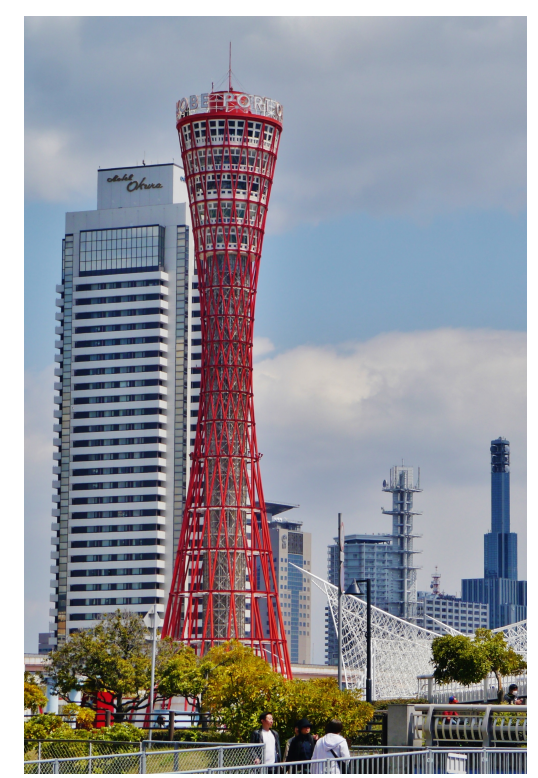

Figure 1: Kobe Port Tower in Japan (cf. [1])

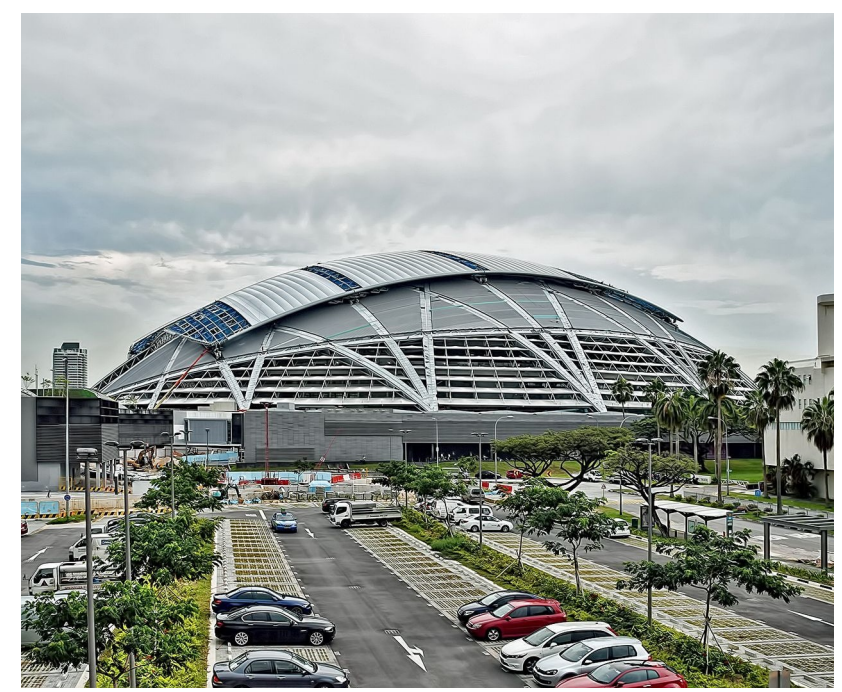

Figure 2: National Stadium in Singapore (cf. [2])

ðOpen Access. @ 2019 E. Koźniewski and A. Borowska, published by De Gruyter. Attribution 4.0 License 
To design structures based on the hyperboloid $S(t, v)$, the coordinates of points of other surfaces that approximate the hyperboloid $S(t, v)$ are necessary. The hyperboloid $S(t, v)$ can be approximated by means of the offset surfaces, a hyperboloid of similar parameters or another surface with the desired properties. The paper provides a convenient methodology for acquiring the angles and coordinates of points of various surfaces that approximate the one-sheet (or two-sheet) hyperboloid surface.

Section 3 suggests the construction of a reinforced hyperboloid-shaped double-layer dome. Section 4 presents a proposal for a reinforced cooling tower structure. Sections 2, 3.1, 3.2 and 4.1 provide mathematical formulas defining the angles and coordinates of points of different surfaces approximating the hyperboloid. The interesting offset surfaces (offset curves) are described in [3-5]. Section 5 contains examples of the applications of the given geometric method.

\section{Mathematical formulas}

Let $c(t)=(x(t), y(t))(t \in[\alpha, \beta])$ be a parametric representation of a planar curve (we write down functions $x(t), y(\mathrm{t})$ as $\left.x_{t}, y_{t}\right)$. The normal vector to the curve $c(t)$ at the point $P\left(x_{t}, y_{t}\right)$ is as follows $\mathrm{n}=\left[-y_{t}^{\prime}, x_{t}^{\prime}\right]$. The unit normal vector at the point $P\left(x_{t}, y_{t}\right)$ is of the form (cf. [6], p. 335)

$$
\mathrm{n}_{\mathrm{ver}}=\left[-y_{t}^{\prime}, x_{t}^{\prime}\right] / \sqrt{\left(x_{t}^{\prime}\right)^{2}+\left(y_{t}^{\prime}\right)^{2}} .
$$

For a smooth planar curve $c$, we define an offset curve $c_{d}$ at distance $d$ in the following way. On each curve normal, we mark the two points that are at distance $d$ from the curve $c$. The set of all of these points forms the offset $c_{d}=\left(c_{d}^{\prime} \cup c_{d}^{\prime \prime}\right)$ (cf. [6], p. 335). The offset $c_{d}(t)$ at distance $d$ to $c(t)$ is obtained as (cf. [6], p. 335) $c_{d}(t)=c(t) \pm d \mathrm{n}_{\text {ver }}(t)$. The curve $c$ and its offset curves $c_{d}^{\prime}$ and $c_{d}^{\prime \prime}$ are not always of the same type.

Let us assume that $P$ is any point on the hyperbola $c_{h}$ and $l$ is the normal line to $c_{h}$ at the point $P$. Points $P_{1}$ and $P_{2}$ lie on the normal lat distance $d$ from $P . Q_{1}, Q_{2}$ are the in tersection points of the normal line $l$ with hyperbolas $c_{h 1}$ and $c_{h 2}$ respectively. Non-zero distances $d_{P 1 Q 1}=\left|\overline{P_{1} Q_{1}}\right|$ and $d_{P 2 Q 2}=\left|\overline{P_{2} Q_{2}}\right|$ mean that hyperbolas $c_{h 1}$ and $c_{h 2}$ do not keep a constant distance $d$ relative to the basic hyperbola $c_{h}$ (cf. Figure 3 ).

The $c_{h}(t), c_{h_{1}}(\phi)$ and off $\left(c_{h} ; d\right)$ curves shown in Figure 3 are defined as follows

$$
c_{h}(t): x=a \cosh (t), \quad z=b \sinh (t)
$$

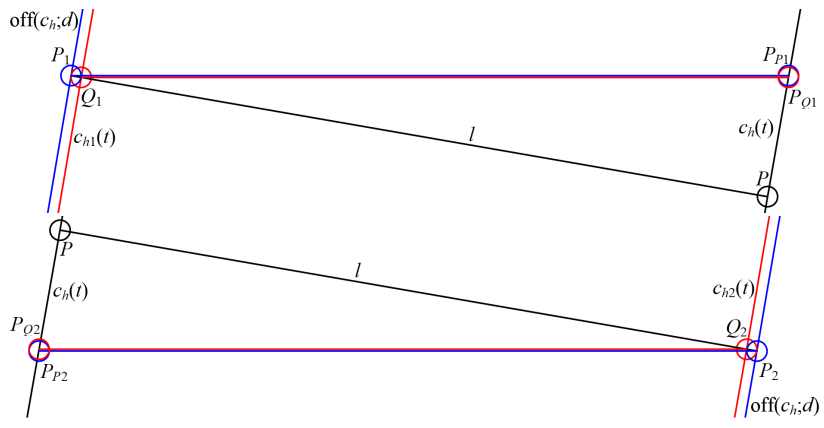

Figure 3: The arrangement of points and curves

(cf. [7], p. 98),

$$
\begin{aligned}
c_{h 1}(\phi): x & =a \cosh (\phi)-d, z=b \sinh (\phi), \\
c_{h 2}(\phi): x & =a \cosh (\phi)+d, z=b \sinh (\phi), \\
\text { off }\left(c_{h} ; d\right) & =c_{h}(t) \pm d \mathrm{n}_{\mathrm{ver}}(t) .
\end{aligned}
$$

Definition 1 (horizontal offset thickness) Let the points $P$, $P_{1}, P_{P 1}\left(z_{P 1}=z_{P P 1}\right)$ and curves $c_{h}, c_{h 1}$, off $\left(c_{h} ; d\right)$ be defined as above (see Figure 3). Horizontal offset thickness measured at height $z=z_{P 1}\left(z_{P 1}\right.$ is arbitrary but fixed) is defined as the length of the section $\left|\overline{P_{1} P_{P 1}}\right|=d_{P 1 P P 1} \geq d$.

Definition 2 (cf. [7], p. 97)

$$
\cosh (t)=\left(e^{t}+e^{-t}\right) / 2, \sinh (t)=\left(e^{t}-e^{-t}\right) / 2 .
$$

\section{Double-layer lattice domes}

In this section, we suggest the use of the two-sheet rotational hyperboloid surface to form double-layer lattice domes. Such roofs of large buildings (stadiums, halls, shopping centres) made of metal bars can be light constructions with considerable spread. The bar structures of the low-profile single-layer domes are particularly susceptible to stability loss [8]. Therefore, a more advantageous solution is the construction of double-layer structures connected by bars [9]. We provide a convenient method for determining the angles and coordinates of points of various surfaces (also the offset surfaces) approximating the twosheet hyperboloid surface.

\subsection{The coordinates of points $\boldsymbol{P}_{1}$ and $\boldsymbol{P}_{\mathbf{2}}$}

Let us take the parametric equations of the hyperbola $C_{h}(t): x=a \sinh (t), z=-b \cosh (t)$. We can assume that $t \geq 0$, because the graph of the curve $C_{h}(t)$ is symmetrical about the $Z$ axis. The coordinates of points $P_{1}$ and $P_{2}$ lying 
on the normal $l$ to the hyperbola $C_{h}(t)$ (at the point $P\left(x_{t}\right.$, $\left.z_{t}\right)$ ) and distant from $P$ by the length $d$ were determined using the following equation of the offset curves off $\left(C_{h}(t)\right.$; d)

$$
\begin{aligned}
\operatorname{off}\left(C_{h}(t) ; d\right) & :[X, Z]= \\
& =\left[x_{t}, z_{t}\right] \pm \frac{d[b \sinh (t), a \cosh (t)]}{\sqrt{a^{2} \cosh ^{2}(t)+b^{2} \sinh ^{2}(t)}}
\end{aligned}
$$

for $x_{t}=a \sinh (t), z_{t}=-b \cosh (t)$. From here we obtain the coordinates of points $P_{1}$ and $P_{2}$

$$
\begin{aligned}
& x_{P 1}=x_{t}-\frac{d b \sinh (t)}{\sqrt{a^{2} \cosh ^{2}(t)+b^{2} \sinh ^{2}(t)}}, \\
& z_{P 1}=z_{t}-\frac{d a \cosh (t)}{\sqrt{a^{2} \cosh ^{2}(t)+b^{2} \sinh ^{2}(t)}}, \\
& x_{P 2}=x_{t}+\frac{d b \sinh (t)}{\sqrt{a^{2} \cosh ^{2}(t)+b^{2} \sinh ^{2}(t)}}, \\
& z_{P 2}=z_{t}+\frac{d a \cosh (t)}{\sqrt{a^{2} \cosh ^{2}(t)+b^{2} \sinh ^{2}(t)}}
\end{aligned}
$$

for $x_{t}=a \sinh (t), z_{t}=-b \cosh (t)$.

\subsection{The coordinates of the point $Q_{1}$}

Let us take the parametric equations of the hyperbola $C_{h 1}(\phi): x=a \sinh (\phi), z=-b \cosh (\phi)-d$. The coordinates of the point $Q_{1}$ (the intersection of the normal line $l$ to the hyperbola $C_{h}(t)$ at the point $P\left(x_{t}, z_{t}\right)$ with the hyperbola $\left.C_{h 1}(\phi)\right)$ were determined as follows. We can assume that $t, \phi \geq 0$. The normal vector to the curve $C_{h}(t)$ at the point $P$ is of the form $\mathrm{n}=[b \sinh (t), a \cosh (t)]$. The parametric equations of the normal line $l$ to the hyperbola $C_{h}(t)$ at the point $P$ are as follows (cf. [10], p. 140)

$$
x=\sinh (t)(a-k d b), \quad z=-\cosh (t)(b+k d a) .
$$

Let us set the parameter $k$ giving the intersection points of the line $l$ with the hyperbola $C_{h 1}(\phi)$.

$$
\begin{aligned}
& x=\sinh (t)(a-k d b)=a \sinh (\phi), \\
& z=-\cosh (t)(b+k d a)=-(b \cosh (\phi)+d) .
\end{aligned}
$$

Let us determine the parameter $k$ from the second equation and put it in the first equation.

$$
\begin{aligned}
& k=\frac{b}{d a}\left(\frac{\cosh (\phi)}{\cosh (t)}-1\right)+\frac{1}{a \cosh (t)} \\
& a \sinh (t)+\frac{b^{2}}{a} \sinh (t)-\frac{b^{2} \cosh (\phi) \sinh (t)}{a \cosh (t)}-\frac{b d \sinh (t)}{a \cosh (t)}=
\end{aligned}
$$

$$
\begin{aligned}
& =a \sinh (\phi) \quad / \cdot a \cosh (t) \\
& a^{2} \sinh (\phi) \cosh (t)+b^{2} \cosh (\phi) \sinh (t)= \\
& =\sinh (t) \cosh (t)\left(a^{2}+b^{2}\right)-d b \sinh (t)
\end{aligned}
$$

Hence and from definition 2 we have

$$
\begin{aligned}
& a^{2} \cosh (t)\left(e^{\phi}-e^{-\phi}\right) / 2+b^{2} \sinh (t)\left(e^{\phi}+e^{-\phi}\right) / 2= \\
& =\sinh (t) \cosh (t)\left(a^{2}+b^{2}\right)-d b \sinh (t)
\end{aligned}
$$

Hence

$$
A e^{2 \phi}+B e^{\phi}+C=0
$$

where

$A=a^{2} \cosh (t)+b^{2} \sinh (t)$,

$B=-2 \sinh (t)\left(\cosh (t)\left(a^{2}+b^{2}\right)-d b\right)$,

$C=b^{2} \sinh (t)-a^{2} \cosh (t)$.

Let us denote $E=e^{\phi}$. Then for $\Delta=B^{2}-4 A C>0$ we have $E_{1}=(-B+\sqrt{\Delta}) / 2 A$ and $E_{2}=(-B-\sqrt{\Delta}) / 2 A$. Hence $\phi_{1}=$ $\ln E_{1}$ and $\phi_{2}=\ln E_{2}$. Finally we obtain the parameter $k$ for the point $Q_{1}$

$$
\begin{aligned}
k & =k_{1}=\frac{b}{d a}\left(\frac{\cosh \left(\phi_{1}\right)}{\cosh (t)}-1\right)+\frac{1}{a \cosh (t)}, \\
\phi_{1} & =\ln E_{1}=\operatorname{arsinh}\left(\sinh (t)\left(1-\frac{k_{1} d b}{a}\right)\right) .
\end{aligned}
$$

Lemma 1 (cf. [5], Lemma 3, p. 46)

Let us denote $k_{P 1}=1 / \sqrt{b^{2} \sinh ^{2}(t)+a^{2} \cosh ^{2}(t)}$.

(a) The coordinates of the points $P, P_{1}$ and $Q_{1}$ can be determined using the parametric equations (6) of the normal line $l$ to the hyperbola $C_{h}(t)$ at the point $P\left(x_{t}, z_{t}\right)$ (we also write down $P\left(x_{P}, z_{P}\right)$ ) for the parameter $k$ equal respectively $k=0, k=k_{P 1}$ (cf. (5)) and $k=k_{1}$ (cf. (7)).

(b) $d_{P Q 1} \leq d$ iff $k_{1} \leq k_{P 1}$.

The property (b) results from the fact that points $P, P_{1}$ and $Q_{1}$ lie on the normal $l$.

Figure 4 shows the two-sheet rotational hyperboloid $S_{h}(t, v)$ (the red surface), the two-sheet rotational hyperboloid $S_{h 1}(\phi, v)$ (the bright blue surface) and the hyperboloid offset surface off $\left(S_{h} ; d\right)$ at distance $d$ (the dark blue surface).

$$
\begin{aligned}
& S_{h}(t, v): \quad x=(a \sinh (t)) \cos (v), \\
& y=(a \sinh (t)) \sin (v), \quad z=-b \cosh (t), \\
& S_{h 1}(\phi, v): \quad x=(a \sinh (\phi)) \cos (v), \\
& y=(a \sinh (\phi)) \sin (v), \quad z=-b \cosh (\phi)-d, \\
& \operatorname{off}\left(S_{h} ; d\right): \quad x=\sinh (t)\left(a-d b k_{P 1}\right) \cos (v),
\end{aligned}
$$




$$
\begin{aligned}
& y=\sinh (t)\left(a-d b k_{P_{1}}\right) \sin (v), \\
& z=-\cosh (t)\left(b+d a k_{P_{1}}\right),
\end{aligned}
$$

where $k_{P 1}=1 / \sqrt{b^{2} \sinh ^{2}(t)+a^{2} \cosh ^{2}(t)}$.

Figure 5 shows a fragment of the double-layer lattice dome of the shape based on the two-sheet rotational hyperboloid. The black lattice is shaped by the hyperboloid $S_{h}(t, v)(a=30, b=40)$, the blue lattice is formed by the offset surface off $\left(S_{h} ; d\right)(d=6)$ and the pink lattice is a part

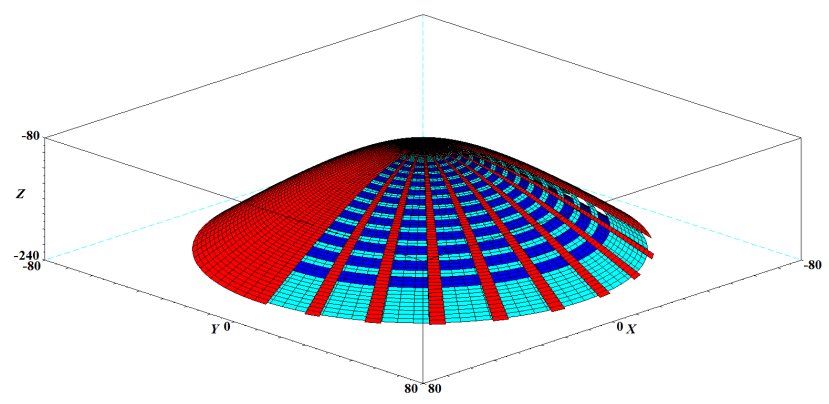

Figure 4: Fragment of the (red) two-sheet hyperboloid $S_{h}(t, v)$, the (bright blue) two-sheet hyperboloid $S_{h 1}(\phi, v)$ and the (blue) hyperboloid offset surface off $\left(S_{h} ; d\right)$

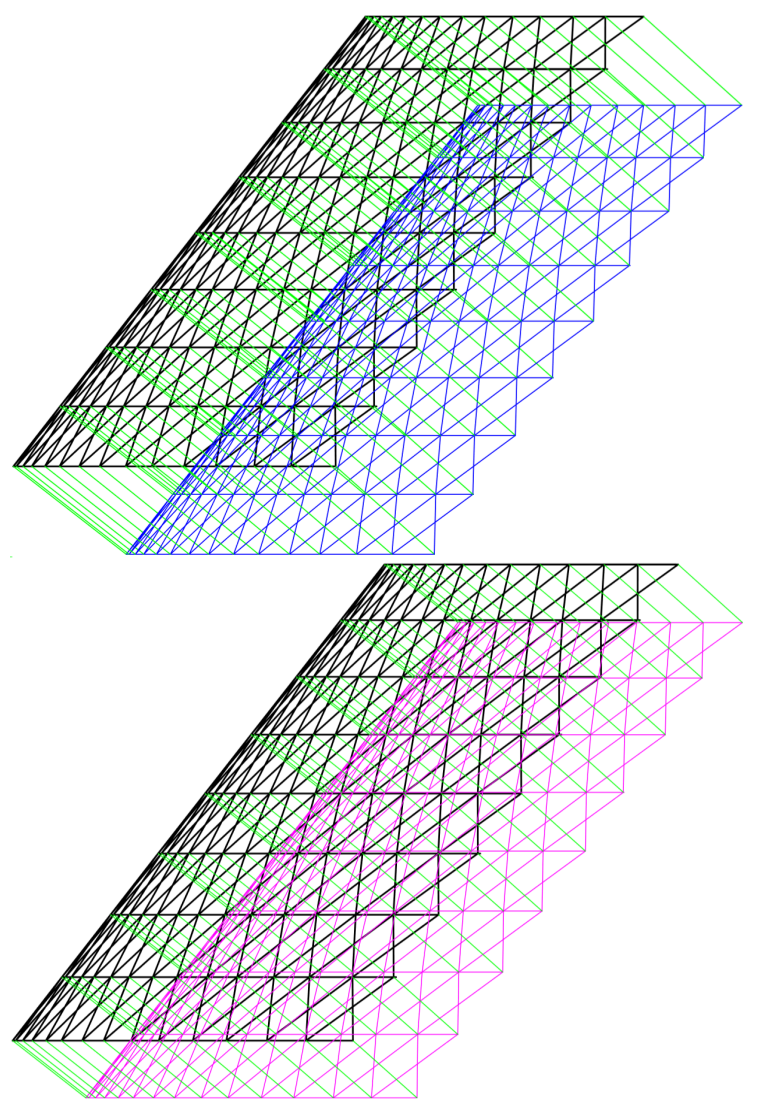

Figure 5: Fragment of the double-layer lattice dome of the shape based on the two-sheet rotational hyperboloid

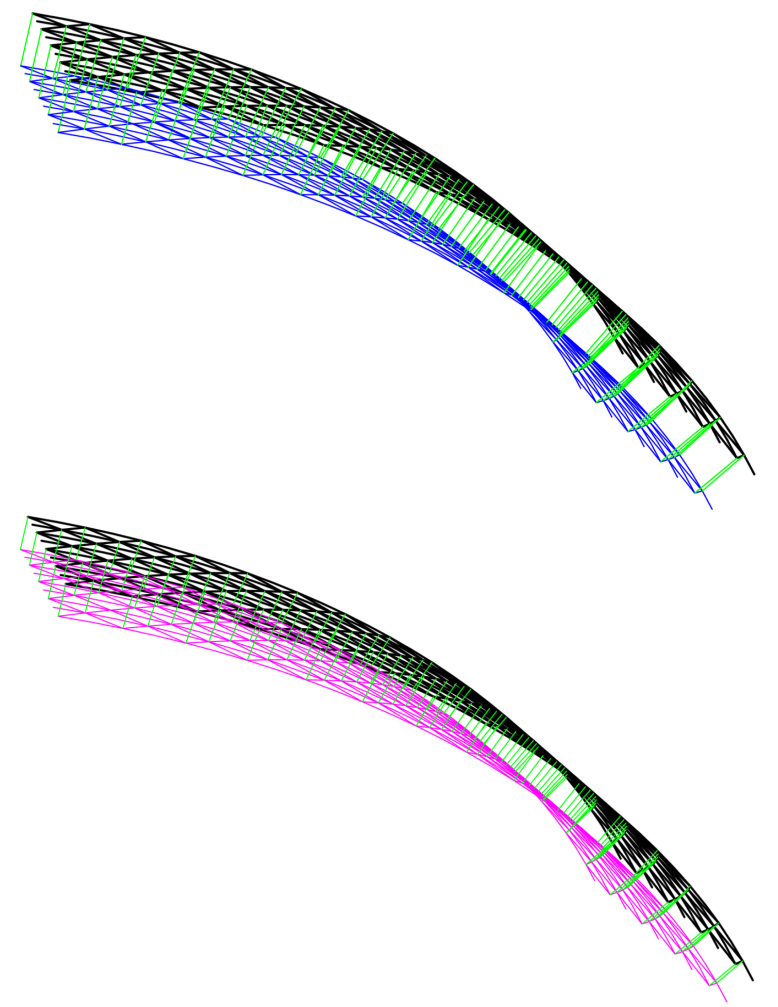

Figure 6: Fragment of the double-layer lattice dome of the shape based on the two-sheet rotational hyperboloid

of the hyperboloid $S_{h 1}(\phi, v)$. The bars connecting the two lattice surfaces are perpendicular to the outer surface. The given formulas allow designers to use any lattice pattern.

\section{Cooling towers}

A cooling tower is a device used to cool industrial water in those energy and industrial plants that do not have the possibility to make use of water from a river, lake or sea. Cooling towers are equipped with a very high (usually) reinforced concrete chimney. The chimney wall is a thin shell in the shape (from outside and inside) of the one-sheet rotational hyperboloid. This chimney construction is rigid and resistant to bending. Such a design guarantees the possibility of obtaining a large diameter and height. Thanks to the high chimney and the heated water, the so-called chimney effect is obtained.

Figure 7 shows the model of the chimney wall. The one-sheet hyperboloid surface $s_{h}(t, v)$ modelling the outer part of the chimney wall is coloured bright blue. The onesheet hyperboloid surface $s_{h_{1}}(\phi, v)$ modelling the inner part of the chimney wall is coloured red. The offset surface $\operatorname{off}\left(s_{h} ; d\right)$ at distance $d$ to the outer part of the chimney wall 


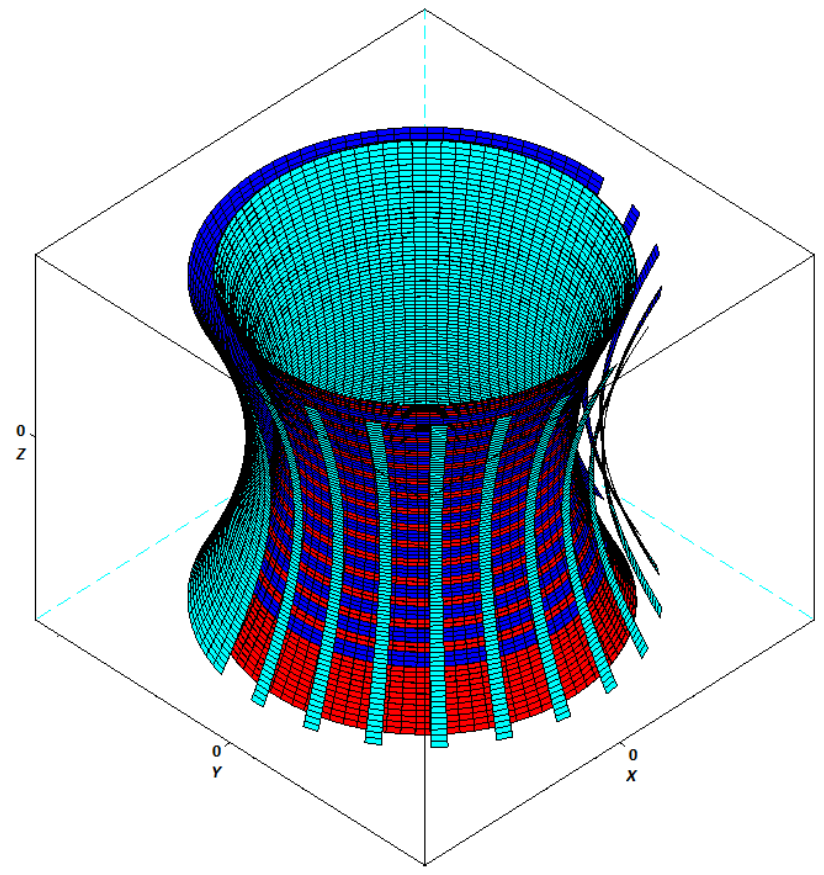

Figure 7: Wall model of a cooling tower

is coloured dark blue. The use of the offset surface (i.e. horizontal offset thickness) could reinforce the structure of the chimney wall. The wall would be more resistant to wind force. The surfaces $s_{h}(t, v)$ and $s_{h 1}(\phi, v)$ were cut out to show the next layer (see Figure 7).

$$
\begin{aligned}
s_{h}(t, v): \quad x & =a \cosh (t) \cos (v), \\
y & =a \cosh (t) \sin (v), \quad z=b \sinh (t), \\
s_{h 1}(\phi, v): \quad x & =(a \cosh (\phi)-d) \cos (v), \\
y & =(a \cosh (\phi)-d) \sin (v), \quad z=b \sinh (\phi), \\
\operatorname{off}\left(s_{h} ; d\right) & =s_{h}(t, v)+d \mathrm{n}_{\mathrm{ver}}(t, v),
\end{aligned}
$$

where $\mathrm{n}_{\mathrm{ver}}(t, v)$ is the unit normal vector to the surface $s_{h}(t, v)$ at any point.

\subsection{The coordinates of points $P_{1}, P_{2}, Q_{1}$ and $\mathbf{Q}_{2}$}

Let the points $P, P_{1}, P_{2}, Q_{1}, Q_{2}$ and curves (hyperbolas) $c_{h}(t), c_{h 1}(\phi), c_{h 2}(\phi)$ be defined as in section 2 (see Figure 3, (2), (3)). We can assume that $t, \phi \geq 0$, because the graph of the curve $c_{h}(t)$ (also $c_{h 1}(\phi), c_{h 2}(\phi)$ ) is symmetrical about the $X$ axis. By conducting analogous calculations as in 3.1 we get the coordinates of points $P_{1}$ and $P_{2}$ lying on the normal $l$ to the hyperbola $c_{h}(t)$ (at the point $P\left(x_{t}, z_{t}\right)$ ) and dis- tant from $P$ by the length $d$.

$$
\begin{aligned}
& x_{P 1}=x_{t}-\frac{d b \cosh (t)}{\sqrt{a^{2} \sinh ^{2}(t)+b^{2} \cosh ^{2}(t)}}, \\
& z_{P 1}=z_{t}+\frac{d a \sinh (t)}{\sqrt{a^{2} \sinh ^{2}(t)+b^{2} \cosh ^{2}(t)}}, \\
& x_{P 2}=x_{t}+\frac{d b \cosh (t)}{\sqrt{a^{2} \sinh ^{2}(t)+b^{2} \cosh ^{2}(t)}}, \\
& z_{P 2}=z_{t}-\frac{d a \sinh (t)}{\sqrt{a^{2} \sinh ^{2}(t)+b^{2} \cosh ^{2}(t)}}
\end{aligned}
$$

for $x_{t}=a \cosh (t), z_{t}=b \sinh (t)$.

By conducting analogous calculations as in 3.2 we get the coordinates of the point $Q_{1}$ (and the point $Q_{2}$ ) (the intersection of the normal line $l$ to the hyperbola $c_{h}(t)$ at the point $P\left(x_{t}, z_{t}\right)$ with the hyperbola $c_{h 1}(\phi)$ (with the hyperbola $\left.\left.c_{h 2}(\phi)\right)\right)$.

Lemma 2 (a) The coordinates of the point $Q_{1}$ can be determined using the parametric equations

$$
x=\cosh (t)(a-k d b), \quad z=\sinh (t)(b+k d a)
$$

of the normal line $l$ to the hyperbola $c_{h}(t)$ at the point $P\left(x_{t}, z_{t}\right)$ for the following parameter $k$

$$
\begin{gathered}
k=k_{1}=\frac{a}{d b}\left(1-\frac{\cosh \left(\phi_{1}\right)}{\cosh (t)}\right)+\frac{1}{b \cosh (t)} \\
\phi_{1}=\ln E_{1}=\operatorname{arsinh}\left(\sinh (t)\left(1+\frac{k_{1} d a}{b}\right)\right),
\end{gathered}
$$

where $E_{1}=(-B+\sqrt{\Delta}) / 2 A$ for

$$
\begin{aligned}
& A=a^{2} \sinh (t)+b^{2} \cosh (t), \\
& B=-2 \sinh (t)\left(\cosh (t)\left(a^{2}+b^{2}\right)+d a\right), \\
& C=a^{2} \sinh (t)-b^{2} \cosh (t), \quad \Delta=B^{2}-4 A C .
\end{aligned}
$$

(b) The coordinates of the point $Q_{2}$ can be determined using the parametric equations

$$
x=\cosh (t)(a+k d b), \quad z=\sinh (t)(b-k d a)
$$

of the normal line $l$ to the hyperbola $c_{h}(t)$ at the point $P\left(x_{t}, z_{t}\right)$ for the following parameter $k$

$$
\begin{aligned}
k & =k_{1}=\frac{a}{d b}\left(\frac{\cosh \left(\phi_{1}\right)}{\cosh (t)}-1\right)+\frac{1}{b \cosh (t)} \\
\phi_{1} & =\ln E_{1}=\operatorname{arsinh}\left(\sinh (t)\left(1-\frac{k_{1} d a}{b}\right)\right),
\end{aligned}
$$

where $E_{1}=(-B+\sqrt{\Delta}) / 2 A$ for

$$
\begin{aligned}
& A=a^{2} \sinh (t)+b^{2} \cosh (t), \\
& B=-2 \sinh (t)\left(\cosh (t)\left(a^{2}+b^{2}\right)-d a\right), \\
& C=a^{2} \sinh (t)-b^{2} \cosh (t), \quad \Delta=B^{2}-4 A C .
\end{aligned}
$$




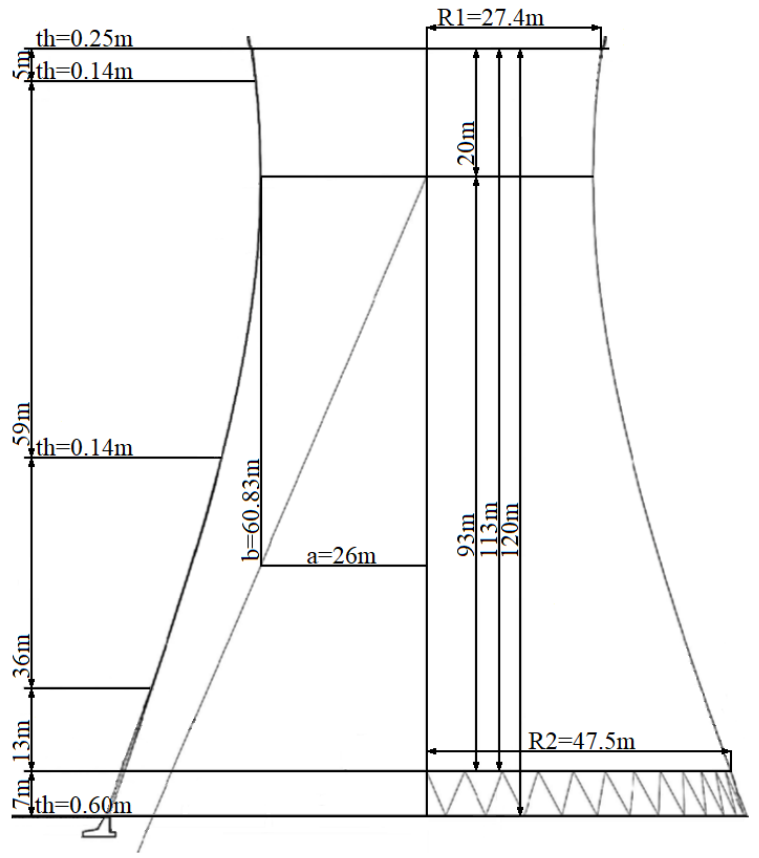

Figure 8: Diagram of the Rybnik chimney (cf. [11, 12])

\subsection{Variability of the thickness of the cooling tower shell}

The deviation of the chimney wall thickness from the horizontal offset thickness has been analyzed. The structure under investigation was the chimney of the Rybnik cooling tower. The outer side of the chimney of the Rybnik cooling tower has the shape of the hyperboloid $S R_{h}(t, v)$. Parameters of the Rybnik chimney are shown in Figure $8(a=26 \mathrm{~m}$, $b=60.83 \mathrm{~m}$, constant shell thickness $d=0.14 \mathrm{~m}$, radius of the bottom base $R_{2}=47.5 \mathrm{~m}$, radius of the upper base $\mathrm{R}_{1}=27.4 \mathrm{~m}$, chimney height $z=93+20 \mathrm{~m}$ ). Figure 8 also shows the variability of the chimney shell thickness. The first ring from the bottom is $0.6 \mathrm{~m}$ thick. Next, on the $13 \mathrm{me}$ tres section, the thickness of the shell decreases to $0.18 \mathrm{~m}$ according to the hyperbolic function. On the next 36 metres section the thickness decreases linearly to $0.14 \mathrm{~m}$. At the next stage $(59 \mathrm{~m})$ the thickness is constant, equal to $0.14 \mathrm{~m}$. Over the last $5 \mathrm{~m}$ the thickness increases linearly to $0.25 \mathrm{~m}$. We assume that the inside of the chimney (on the 59 metres section) has the shape of the hyperboloid $S R_{h 1}(\phi, v)$.

$$
\begin{aligned}
S R_{h}(t, v): \quad x=26 \cosh (t) \cos (v), \\
\quad y=26 \cosh (t) \sin (v), \quad z=60.83 \sinh (t), \\
S R_{h 1}(\phi, v): \quad x=(26 \cosh (\phi)-d) \cos (v), \\
y=(26 \cosh (\phi)-d) \sin (v), \\
z=60.83 \sinh (\phi) .
\end{aligned}
$$

The measurements of the shape of the outer surface of the Rybnik chimney made in 1991 and 2010 showed the execution of the object with numerous deviations from the ideal geometry of the one-sheet rotational hyperboloid. The results obtained in 2010 gave extreme deviation values (to the interior of the shell $-1.124 \mathrm{~m}$, outside the shell $+0.788 \mathrm{~m}$ ) (cf. [11]).

The measurements made in the last 19 years have shown a very disturbing increment of displacements in extreme cases reaching values of $0.3-0.4 \mathrm{~m}$ (with a tendency to move towards the interior in the lower part of the shell and outside in the upper one). The tests showed a clear threat to the load-bearing capacity and stability of the object (cf. [11]).

The method for determining the wall thickness of the cooling tower has been given. The thickness of the chimney wall is measured along the normal line $l$ to the external surface of the chimney passing through the point $P$. More specifically, it was assumed that the external surface (the inner surface) of the chimney was shaped as the hyperboloid surface $S R_{h}(t, v)$ (respectively as the hyperboloid surface $\left.S R_{h 1}(\phi, v)\right)$. The formula specifying distance $d_{P Q 1}=\overline{\left|P Q_{1}\right|}$ (measured along the normal $l$ to the surface $S R_{h}(t, v)$, between the point $P$ and the point $Q_{1}$ (the intersection of the normal $l$ with the hyperboloid surface $\left.S R_{h 1}(\phi, v)\right)$ ) was given. Because both hyperboloids are surfaces of revolution, they were replaced with hyperbolas $c_{h}(t)$ and $c_{h 1}(\phi)$ on the $X Z$ plane. Because the hyperbolas graphs are symmetrical about the $X$ axis, it was assumed that $t, \phi \geq 0$. Points and curves are defined as in Figure 3.

Test 1 A numerical analysis was carried out for the problem described above (see Table 1).

The distances $d_{P Q 1}$ were measured for points $P\left(x_{P}, z_{P}\right)$, where $z_{P}=0,10, \ldots, 100 \mathrm{~m}$. The distance $d_{P Q 1}$ decreases with the increase of $z_{P}$ and at the height $z_{P}=100 \mathrm{~m} d_{P Q 1}=0.939 d$.

In order to strengthen the chimney wall (at the design stage) in the part where the thickness of the shell is smallest, it is proposed to design a wall with the horizontal offset thickness. Such a construction will be more resistant to wind force. For this purpose, it is enough to shape the inside of the chimney using the hyperboloid offset surface off $\left(S R_{h} ; d\right)$

$$
\begin{aligned}
\operatorname{off}\left(S R_{h} ; d\right): x & =\cosh (t)\left(a-d b k_{P 1}\right) \cos (v), \\
y & =\cosh (t)\left(a-d b k_{P 1}\right) \sin (v), \\
z & =\sinh (t)\left(b+d a k_{P 1}\right),
\end{aligned}
$$

where $k_{P 1}=1 / \sqrt{a^{2} \sinh ^{2}(t)+b^{2} \cosh ^{2}(t)}$. 
Table 1: Coordinates of points $P$ and $Q_{1}$, the angle $t$ (for the point $P$ ) and $\phi$ (for the point $Q_{1}$ ), coefficient $k$ and distance $d_{P Q 1}$ measured at successive heights of the Rybnik chimney

\begin{tabular}{cccc}
\hline$x_{P} / z_{P}$ & $t / \phi$ & $k / d_{P Q 1}$ & $x_{Q 1} / z_{Q 1}$ \\
\hline$x_{P}=26$ & $t=0^{\circ}$ & $k=0.016439$ & $x_{Q 1}=25.86$ \\
$z_{P}=0$ & $\phi 0^{\circ}$ & $d_{P Q 1}=0.14(100 \%)$ & $z_{Q 1}=0$ \\
$x_{P}=26.3490$ & $t=9^{\circ} 22^{\prime} 37^{\prime \prime}$ & $k=0.016144$ & $x_{Q 1}=26.2096$ \\
$z_{P}=10$ & $\phi=9^{\circ} 23^{\prime} 10^{\prime \prime}$ & $d_{P Q 1}=0.1397(99.8 \%)$ & $z_{Q 1}=10.0097$ \\
$x_{P}=27.3692$ & $t=18^{\circ} 30^{\prime} 51^{\prime \prime}$ & $k=0.015343$ & $x_{Q 1}=27.2317$ \\
$z_{P}=20$ & $\phi=18^{\circ} 31^{\prime} 50^{\prime \prime}$ & $d_{P Q 1}=0.1388(99.1 \%)$ & $z_{Q 1}=20.0184$ \\
$x_{P}=28.9900$ & $t=27^{\circ} 13^{\prime} 17^{\prime \prime}$ & $k=0.014235$ & $x_{Q 1}=28.8548$ \\
$z_{P}=30$ & $\phi=27^{\circ} 14^{\prime} 34^{\prime \prime}$ & $d_{P Q 1}=0.1376(98.3 \%)$ & $z_{Q 1}=30.0255$ \\
$x_{P}=31.1175$ & $t=35^{\circ} 23^{\prime} 1^{\prime \prime}$ & $k=0.013017$ & $x_{Q 1}=30.9849$ \\
$z_{P}=40$ & $\phi=3^{\circ} 24^{\prime} 29^{\prime \prime}$ & $d_{P Q 1}=0.1363(97.3 \%)$ & $z_{Q 1}=40.0311$ \\
$x_{P}=33.6559$ & $t=42^{\circ} 57^{\prime} 22^{\prime \prime}$ & $k=0.011828$ & $x_{Q 1}=33.5255$ \\
$z_{P}=50$ & $\phi=42^{\circ} 58^{\prime} 55^{\prime \prime}$ & $d_{P Q 1}=0.1351(96.5 \%)$ & $z_{Q 1}=50.0354$ \\
$x_{P}=36.5196$ & $t=49^{\circ} 56^{\prime} 39^{\prime \prime}$ & $k=0.010736$ & $x_{Q 1}=36.3911$ \\
$z_{P}=60$ & $\phi=49^{\circ} 58^{\prime} 12^{\prime \prime}$ & $d_{P Q 1}=0.1341(95.8 \%)$ & $z_{Q 1}=60.0385$ \\
$x_{P}=39.6380$ & $t=56^{\circ} 22^{\prime} 56^{\prime \prime}$ & $k=0.009766$ & $x_{Q 1}=39.5112$ \\
$z_{P}=70$ & $\phi=56^{\circ} 24^{\prime} 27^{\prime \prime}$ & $d_{P Q 1}=0.1332(95.1 \%)$ & $z_{Q 1}=70.0409$ \\
$x_{P}=42.9559$ & $t=62^{\circ} 19^{\prime} 4^{\prime \prime}$ & $k=0.008918$ & $x_{Q 1}=42.8304$ \\
$z_{P}=80$ & $\phi=62^{\circ} 20^{\prime} 31^{\prime \prime}$ & $d_{P Q 1}=0.1325(94.6 \%)$ & $z_{Q 1}=80.0427$ \\
$x_{P}=46.4303$ & $t=67^{\circ} 48^{\prime} 5^{\prime \prime}$ & $k=0.008180$ & $x_{Q 1}=46.3059$ \\
$z_{P}=90$ & $\phi=67^{\circ} 49^{\prime} 28^{\prime \prime}$ & $d_{P Q 1}=0.1320(94.3 \%)$ & $z_{Q 1}=90.0440$ \\
$x_{P}=50.0288$ & $t=72^{\circ} 52^{\prime} 56^{\prime \prime}$ & $k=0.007538$ & $x_{Q 1}=49.9053$ \\
$z_{P}=100$ & $\phi=72^{\circ} 54^{\prime} 16^{\prime \prime}$ & $d_{P Q 1}=0.1315(93.9 \%)$ & $z_{Q 1}=100.0451$ \\
\hline
\end{tabular}

Test 2 A numerical analysis was carried out for the following problem. At the following heights $z_{P P 1}=0,10$, $\ldots, 100 \mathrm{~m}$ of the chimney (see the point $P_{P 1}\left(x_{P P 1}, z_{P P 1}\right)$ in Figure 3) the horizontal offset thickness of the chimney wall was determined. I.e. for consecutive values $z_{P 1}=z_{P P 1}$ the coordinates of points $P_{1}, P_{P 1}$ and distance $d_{P 1 P P 1}=\overline{\left|P_{1} P_{P 1}\right|}$ were calculated. Because the hyperboloid $S R_{h}(t, v)$ and the offset off( $\left(S R_{h} ; d\right)$ are surfaces of revolution, they were replaced with hyperbola $c_{h}(t)$ and offset $\operatorname{off}\left(c_{h} ; d\right)$ on the $X Z$ plane. Because the graphs of both these curves are symmetrical about the $X$ axis, it was assumed that $t, \phi \geq 0$. Points and curves are defined as in Figure 3.

Method. For each value $i=0,10, \ldots, 100$ such an angle $t$ was determined that $z_{P 1}(t)=i$. Because $z_{P 1}(t)=$ $z_{P P 1}(t)$, the coordinates of the point $P_{P 1}$ can be determined as follows $x_{P P 1}=a \cosh (\alpha), z_{P P 1}=b \sinh (\alpha)$, where $\alpha=\operatorname{arsinh}\left(z_{P 1} / b\right)$. Additionally, the point $Q_{1}\left(x_{Q 1}, z_{Q 1}\right)$ was determined for the angle $\phi$ (cf. (10)). Table 2 also gives the distance $d_{P Q 1} \leq d$ (when the inner wall is shaped as the surface $S R_{h 1}$ ) and the horizontal offset thickness $d_{P 1 P P 1} \geq d$ (when the inner wall is shaped as the offset surface off $\left.\left(S R_{h} ; d\right)\right)$. The distance $d_{P 1 P P 1}$ increases with increasing $z_{P 1}$.

\section{Applications of the given method}

The given geometric method can be used, among other things, for modelling hyperboloid objects in architecture and civil engineering design. Its advantages will be presented using examples of domes and elevations designed with a hyperboloid shape.

The geometry of the base surface of the dome (roof) has a decisive influence on the majority of its construction features: load-bearing capacity, rigidity, simplicity of execution, aesthetics. Therefore, at present mainly geodesic lattice domes are used (low dead weight of the structure with relatively high load capacity [13]) (cf. [14]). Since the offset surfaces of the sphere are spheres, it is easy to obtain surfaces approximating the base sphere. However, for more representative architectural objects, designers propose more glamorous roofs (Sydney Opera House, Wanda Metropolitano (Madrit), Yas Island Marina Hotel 
Table 2: Coordinates of points $P, P_{P 1}, P_{1}$ and $Q_{1}$, the angle $t$ (for the point $P$ ), $\phi$ (for the point $Q_{1}$ ), $\alpha$ (for the point $P_{P 1}$ ) and distances $d_{P Q 1}$ and $d_{P 1 P P 1}$ measured at successive heights of the Rybnik chimney

\begin{tabular}{|c|c|c|c|}
\hline$x_{P} / x_{P P 1} / x_{P 1} / x_{Q 1}$ & $z_{P} / z_{P P 1} / z_{P 1} / z_{Q 1}$ & $t / \phi / \alpha$ & $d_{P Q 1} / d_{P 1 P P 1}$ \\
\hline$x_{P}=26$ & $z_{P}=0$ & $t=0^{\circ}$ & $d_{P Q 1}=0.14$ \\
\hline$x_{P P 1}=26$ & $z_{P P 1}=0$ & $\phi=0^{\circ}$ & $(100 \%)$ \\
\hline$x_{P 1}=25.86$ & $z_{P 1}=0$ & $\alpha=0^{\circ}$ & $d_{P 1 P P 1}=0.14$ \\
\hline$x_{Q 1}=25.86$ & $z_{Q 1}=0$ & & \\
\hline$x_{P}=27.3668$ & $z_{P}=19.9815$ & $t=18^{\circ} 29^{\prime} 51^{\prime \prime}$ & $d_{P Q 1}=0.1388$ \\
\hline$x_{P P 1}=27.3692$ & $z_{P P 1}=20$ & $\phi=18^{\circ} 30^{\prime} 50^{\prime \prime}$ & (99.1\%) \\
\hline$x_{P 1}=27.2280$ & $z_{P 1}=20$ & $\alpha=18^{\circ} 30^{\prime} 51^{\prime \prime}$ & $d_{P 1 P P 1}=0.1412$ \\
\hline$x_{Q 1}=27.2292$ & $z_{Q 1}=19.9998$ & & \\
\hline$x_{P}=31.1100$ & $z_{P}=39.9680$ & $t=35^{\circ} 21^{\prime} 30^{\prime \prime}$ & $d_{P Q 1}=0.1363$ \\
\hline$x_{P P 1}=31.1175$ & $z_{P P 1}=40$ & $\phi=35^{\circ} 22^{\prime} 58^{\prime \prime}$ & (97.3\%) \\
\hline$x_{P 1}=30.9737$ & $z_{P 1}=40$ & $\alpha=35^{\circ} 23^{\prime} 1^{\prime \prime}$ & $d_{P 1 P P 1}=0.1438$ \\
\hline$x_{Q 1}=30.9773$ & $z_{Q 1}=39.9991$ & & \\
\hline$x_{P}=36.5075$ & $z_{P}=59.9598$ & $t=49^{\circ} 55^{\prime} 2^{\prime \prime}$ & $d_{P Q 1}=0.1341$ \\
\hline$x_{P P 1}=36.5196$ & $z_{P P 1}=60$ & $\phi=49^{\circ} 56^{\prime} 35^{\prime \prime}$ & (95.8\%) \\
\hline$x_{P 1}=36.3734$ & $z_{P 1}=60$ & $\alpha=49^{\circ} 56^{\prime} 39^{\prime \prime}$ & $d_{P 1 P P 1}=0.1462$ \\
\hline$x_{Q 1}=36.3790$ & $z_{Q 1}=59.9983$ & & \\
\hline$x_{P}=42.9405$ & $z_{P}=79.9549$ & $t=62^{\circ} 17^{\prime} 31^{\prime \prime}$ & $d_{P Q 1}=0.1325$ \\
\hline$x_{P P 1}=42.9559$ & $z_{P P 1}=80$ & $\phi=62^{\circ} 18^{\prime} 59^{\prime \prime}$ & $(94.6 \%)$ \\
\hline$x_{P 1}=42.8080$ & $z_{P 1}=80$ & $\alpha=62^{\circ} 19^{\prime} 4^{\prime \prime}$ & $d_{P 1 P P 1}=0.1479$ \\
\hline$x_{Q 1}=42.8150$ & $z_{Q 1}=79.9976$ & & \\
\hline$x_{P}=50.0113$ & $z_{P}=99.9520$ & $t=72^{\circ} 51^{\prime} 32^{\prime \prime}$ & $d_{P Q 1}=0.1315$ \\
\hline$x_{P P 1}=50.0288$ & $z_{P P 1}=100$ & $\phi=72^{\circ} 52^{\prime} 51^{\prime \prime}$ & (93.9\%) \\
\hline$x_{P 1}=49.8798$ & $z_{P 1}=100$ & $\alpha=72^{\circ} 52^{\prime} 56^{\prime \prime}$ & $d_{P 1 P P 1}=0.1490$ \\
\hline$x_{Q 1}=49.8878$ & $z_{Q 1}=99.9971$ & & \\
\hline
\end{tabular}

(Abu Dhabi), Mercedes Benz Stadium (Atlanta), Khalifa International Stadium (Qatar).

In order to shape a lattice dome (or a roof) it is necessary to define (in a mathematical way) the surface on which the coating will be stretched. The proposed method provides simple mathematical formulas for angles and coordinates of points of various surfaces approximating the hyperboloid of revolution. Additionally, it allows the designer to adjust the height of the dome in order to ensure its stability. The given method can be used in CAD (ComputerAided Design). (A) Thanks to the given formulas, any element (e.g. a dome, its fragment) of hyperboloid shape can be designed in any programming language (e.g. $\mathrm{Cpp}$ ) and saved in DXF format. The DXF file can be imported into $\mathrm{Au}$ toCAD to get a DWG file (a standard file format for CAD). In this way, the designer can obtain (cf. [15]) in a very short time (at very little cost) a set of complicated digital models (for different parameters). If the object consists of many hyperboloid fragments, in order to make a digital mockup of the whole object, the designer can "assemble" created digital submodels (fragments) instead of scanning a three-dimensional mock-up made by the artist. There are a lot of programs (Digital Project, Rhino-Grasshopper, CATIA, Pro / ENGINEER) that contain parametric design tools and offer designers tools for creating parametric scripts (cf. $[16,17])$. Mainly thanks to them, it was possible to design objects such as Sagrada Familia (Barcelona), Beijing National Stadium (China) (cf. [16]). The reader can see the parametric script for positioning hyperbolas and their placement on a given substrate written (by Burry M.) in Python programming language (cf. $[16,18])$. (B) The possibility of (easy) precise dimensioning of individual elements of a structure with complex geometry can be used for prefabrication using CNC machine (Computerized $\mathrm{Nu}$ merical Control). More precisely, for a 3D model designed in CAD a designer can obtain (using CAM software (Computer Aided Manufacturing)) instructions for a CNC machine [19]. CNC machining allows fast, precise and repeatable execution of elements with a complex shape. Hence, more and more architectural companies are interested in designing objects with a curvilinear geometry. The advantages of assembling prefabricated structures are precise ex- 
ecution, easier transport and assembly. The hyperboloid surface ensures repeatability of elements (e.g. triangular panels) at the same height of the coating.

The given method simplifies the analysis of the approximation of the hyperboloid offset surface off $(S(t, v) ; d)$ by the hyperboloid surface $S_{1}(\phi, v)$. For illustration, the wall thickness of the cooling tower formed by two one-sheet rotational hyperboloids $S R_{h}(t, v)$ and $S R_{h 1}(\phi, v)$ was analyzed (cf. section 4.2). The distance between the two hyperboloid surfaces was measured (along the normal line to the surface $S R_{h}(t, v)$ ). It was shown that the deviation from the constant thickness $d=0.14 \mathrm{~m}$ increases with the increase of the coordinate $z_{P}$. For the cooling tower Rybnik, the largest deviation is $3 \%\left(d_{P Q 1}=0.1358 \mathrm{~m}\right)$ and takes place for $z_{P}=44 \mathrm{~m}(=59 \mathrm{~m}-15 \mathrm{~m}$ (cf. Figure 8$)$ ).

In a similar way, the thickness of the dome formed by the two-sheet rotational hyperboloids $S_{h}(t, v)$ and $S_{h 1}(\phi, v)$ (cf. 3.2) was tested (for the parameter values $a=30, b=40, d=0.4 \mathrm{~m})$. The maximum deviation from the intended thickness $d=0.4 \mathrm{~m}$ (for the dome with a base diameter equal to $30 \mathrm{~m}(40 \mathrm{~m}, 50 \mathrm{~m}$, $60 \mathrm{~m})$ and a height equal to $4.7214 \mathrm{~m}(8.0740 \mathrm{~m}, 12.0684 \mathrm{~m}$, $16.5686 \mathrm{~m})$ ) is $14.00 \%(19.50 \%, 23.86 \%, 27.18 \%)$. The test was repeated for $b=50$. Then the maximum deviation from the intended thickness $d$ (of the dome with a base diameter equal to $30 \mathrm{~m}(40 \mathrm{~m}, 50 \mathrm{~m}, 60 \mathrm{~m})$ and a height equal to $5.9017 \mathrm{~m}(10.0925 \mathrm{~m}, 15.0854 \mathrm{~m}, 20.7107 \mathrm{~m})$ ) was $19.67 \%$ (26.45\%, 31.53\%, 35.24\%).

Similar calculations can help a designer decide whether to use the offset surface and assemble the object from the CNC prefabricates or accept the deviation (or change the values of the hyperboloid parameters).

\section{Summary}

In order to design objects in the shape of the hyperboloid $S(t, v)$, different surfaces approximating this hyperboloid are needed. It is necessary to ensure that the construction has the appropriate thickness (stability). The paper presents the method of determining the angles and coordinates of points of various surfaces approximating the hyperboloid of revolution. Thanks to this method, the designer can easily calculate the distance between the hyperboloid $S(t, v)$ and the surface approximating it along the normal line $l$ passing through the point $P$.

The one-sheet hyperboloid is a doubly ruled surface. It means that hyperboloid shaped constructions can be built with straight steel beams that form a strong structure. The surface approximating the hyperboloid was used to strengthen the wall of the cooling tower (at the design stage). The new mathematical models have been tested and proven in real cases, such as the Rybnik cooling tower chimney, providing real parameters.

A double-layer hyperboloid-shaped lattice dome was proposed as a roof for a large object (e.g. shopping centre). The connection by means of bars of two layers with an approximate shape is intended to strengthen the roof structure. The bar structures of the low-profile single-layer domes are particularly susceptible to stability loss (cf. [8]).

The main advantage of the given method is the simplification of the design process as well as time and cost saving. To design any hyperboloid shaped dome, a student needs a compiler for a programming language $(\operatorname{cost}=0)$ and AutoCAD (for students).

Acknowledgement: The research presented in this paper was founded by the BST S/WI/1/2014 and WZ/WBiIŚ/6/2019.

\section{References}

[1] Figure 1. https://commons.wikimedia.org/wiki/File:Kobe_Kobe_ Port_Tower_\%26_Maritime_Museum_1.jpg.

[2] Figure 2. http://chodor-projekt.net/encyclopedia/najwiekszekopuly.

[3] Koźniewski E., Offsets in geometric creation of roof skeletons with varying slope and cut-and-fill problems in topographic projection, The Journal Biuletyn of Polish Society for Geometry and Engineering Graphics, 2010, vol. 21, 29-35.

[4] Koźniewski M., Thickness analysis of a saddle, The Journal Biuletyn of Polish Society for Geometry and Engineering Graphics, 2016, vol. 28, 25-32.

[5] Borowska A., Approximation of the ellipse offset curves in turbo roundabouts design, The Journal Biuletyn of Polish Society for Geometry and Engineering Graphics, 2018, vol. 31, 43-51.

[6] Pottmann H., Asperl A., Hofer M., Kilian A., Architectural Geometry, 2007, Pennsylvania USA: Bentley Institute Press. Exton.

[7] Leja F., Rachunek różniczkowy i catkowy [Differential and integral calculus], 1973, Warsaw: PWN.

[8] Bysiec D., The investigation of stability of double-layer octahedron-based geodesic domes, Structure and Environment, 2011, vol. 3, No. 3, 30-41.

[9] Mirski J., The investigation of the geometric stability of the selected group of the double-layer bar domes. The 6th ScientificTechnical Conference "Current Scientific and Exploratory Problems in Civil Engineering", The University of Warmia and Mazury, (Olsztyn-Kortowo, Poland), 2003, 225-234.

[10] Grzegorczyk J., Mathematics, 1978, Warsaw: Wydawnictwa Politechniki Warszawskiej.

[11] Mazur J., Zastosowanie nowoczesnych technologii wzmacniania obiektów przemysłwych na przykładzie wzmocnienia płaszcza żelbetowego chłodni kominowej nr 1 w EDF Rybnik SA (Elektrownia Rybnik SA) [Application of modern reinforcement technolo- 
gies for industrial structures shown in exemplary reinforced concrete lining repair at cooling tower no. 1 in EDF Rybnik SA (Rybnik Power Plant SA)]. XXVI Konferencja Naukowo-Techniczna Awarie Budowlane, (Międzyzdroje, Poland), 2013, May 21-24, 1023-1032.

[12] Owczarzy J., Kossowski J., Nieklasyczne zachowanie się modelu hiperboloidalnej chłodni kominowej pod obciążeniem osiowo symetrycznym [A nonclasic behaviour model of hyperbolic shell of cooling tower under axisymmetric loading], Journal of Theoretical and Applied Mechanics, 1981, 2, 19, 225-238.

[13] Radoń U., Zastosowanie metody FORM w analizie niezawodności konstrukcji kratowych podatnych na przeskok [Application method FORM in reliability analysis of node snapping truss structures], Monografie Studia Rozprawy nr M27, 2012, Kielce: Wydawnictwo Politechniki Świętokrzyskiej.

[14] Gayakwad B.R., Hiriyur A., Patil V.V., Arjun K., Lakshmi S., Design considerations for a geodesic dome - a critical review, International Journal of Advances in Science Engineering and Technology, 2018, vol. 6, Iss. 1, 8-13.
[15] Koźniewski E., Geometria odwzorowań inżynierskich powierzchni 05A, Scriptiones Geometrica, 2014, vol. 1, No. 5A, 1-17. https://docplayer.pl/14511873-Geometria-odwzorowaninzynierskich-powierzchnie-05a.html.

[16] Januszkiewicz K., Projektowanie parametryczne oraz parametryczne narzędzia cyfrowe w projektowaniu architektonicznym [Parametric design and parametric digital tools in architectural design], Architecture et Artibus, 2016, vol. 8, No. 3, 43-60.

[17] Stavrić M., Stokić D., Ilić M., Architectural Scale Model in Digital Age - Design Process, Representation and Manufacturing, ECAADE Conference, (Prague, Czech Republic), 2012, September 12, Prague: Faculty of Architecture, 33-41.

[18] Burry M., Scripting Cultures, 2013, Chichester: John Wiley \& Sons Ltd.

[19] Czech-Dudek K., Zastosowanie systemów CAD/CAM w przygotowaniu produkcji [Application systems CAD/CAM in the preparation of production], Mechanik, 2015, 7, 149-158. 